\title{
IEEE 802.11 Goodput Analysis for Mixed Real Time and Data Traffic
}

\author{
Alex Grote, Walter Grote, Rodolfo Feick \\ Electronics Department of Universidad Tecnica Federico Santa Maria, \\ Casilla 110-V, Av. España 1680 Valparaiso, Chile \\ \{agrote,wgrote, feick\}@elo.utfsm.cl
}

\begin{abstract}
An IEEE 802.11 analytical performance evaluation model for ad-hoc WLAN's comprising terminals with different traffic source characteristics is presented. Although some publications address this issue, most of them propose to modify the original standard by some means that will affect the probability of transmission of a device when the network reaches congestion. The approach of this publication is to be able to establish a set of equations such that an intelligent choice of configuration parameters of standard home devices may improve the performance of the wireless network. Actually, two models are presented and compared, a simple one based on stationary behavior of the network assuming collisions have a negligible effect on network performance, and a second model based on a stationary stochastic model of a network, where devices have a packet ready for transmission at all times.
\end{abstract}

\section{Introduction}

Wireless Local Area Networks (WLAN) have expanded massively since the original IEEE 802.11 standard [1] was published in 1999. The amount of devices that use this wireless technology to communicate has increased significantly, a pattern that shows no signs of change since a migration from desktop computers to notebooks can be observed in recent years, of which almost all have a WLAN card preinstalled. User applications have an increasing tendency to require larger bandwidths than it used to. Consequently, amendments to the original standard have been introduced to allow higher data transmission rates (IEEE 802.11b [2] at $11 \mathrm{Mbps}$, IEEE 802.11 a [3] and IEEE 802.11 g [4] at $54 \mathrm{Mbps}$ ). Quality of Service (QoS) has also been incorporated with the recent publication of IEEE 802.11e standard [5], to provide a means to prioritize traffic sent over a wireless LAN to reduce delay for real-time applications as well as other features to enhance the original protocol. Further needs for higher goodput gave way to amendment IEEE $802.11 \mathrm{n}$ [6], which will provide users with at least $100 \mathrm{Mbps}$ wireless connec-

Please use the following format when citing this chapter:

Grote, A.. Grote, W., Feick, R., 2007, in IFIP International Federation for Information Processing, Volume 256, Ilome Networking. $\Lambda \mathrm{I} \wedge$ gha, K., Carcelle, X., Pujolle, G., (Boston: Springer), pp. 15-28. 
tions. These enhancements try to avoid saturation occurrences in wireless networks to provide the connected users with a reliable and fast connection. However, all these enhancements point in the direction of the main focus of this publication: quality assurance for devices operating in IEEE 802.11 wireless networks when operating under different application requirements. The scope of this publication is to be able to establish a set of equations such that an intelligent choice of configuration parameters of standard IEEE.802.11 home devices may improve the performance of the wireless network. Actually, two models are presented and compared, a simple one based on stationary behavior of the network assuming collisions have a negligible effect on network performance, and a second model based on a stationary stochastic model of a network, where devices have a packet ready for transmission at all times. Due to space limitations we will concentrate on ad-hoc networks, but the extension of this analysis to infrastructure network will be quite straightforward.

IEEE 802.11 networks may operate in the ad-hoc mode, where terminals communicate with each other without the need of a centralized access point that is a gateway to a wired backbone network. Random CSMA/CA access is possible by configuring wireless devices of such a network in the DCF (Distributed Coordination Function) mode. DCF employs a backoff mechanism to inhibit transmission once a packet arrives for transmission. The initial value is chosen randomly between 0 and maximum contention window $(C W)$ value. The initial value for the contention window is $C W_{\text {min }}$. This value is increased exponentially by the terminal that is transmitting whenever the transmitted frame collides, thus $C W=2^{\mathrm{i}} \cdot C W_{\text {min }}$ where $i$ represents the number of retransmissions. The counter is decreased from the initial value every time that an idle slot time is registered after the successful transmission, followed by a DIFS time. Once the counter reaches zero the terminal transmits. When a successful transmission takes place, all the terminals of the network that are in the transmission range of the terminal that emits the signal learn how long that transmission will take and pause their backoff counters during that time period.

The access mechanisms defined by DCF are basic access (two-way handshake) and RTS/CTS (four-way handshake). The basic access mode consists of sending a data frame and receiving an acknowledgement (ACK) frame from the destination. RTS/CTS involves reserving the shared medium by the source, sending a RTS (Request To Send) to be responded by a CTS (Clear To Send) packet from the destination, if successfully received, after which the data frame is sent, followed by an ACK. A mixed operation is possible defining a threshold that determines the maximum size of a frame that can be transmitted using basic access. If the frame of user data to be sent is larger than this threshold, a RTS packet is issued by the source, instead of sending the user data frame immediately.

Nowadays terminals present in an ad-hoc network may generate different types of traffic, such as web oriented data traffic, massive file exchanges, real-time voice or video. They also may be configured to set parameter values for the RTS Threshold and initial and maximum contention window settings. The challenge is to be able to come up with a model that will make it possible to optimize network 
performance. We will propose two models aimed to provide solutions for this challenge, based upon recent contributions.

G. Bianchi presents an analytical model to calculate the saturation throughput of a wireless network operating with IEEE 802.11 protocol with a stationary analysis of a stochastic model in [7]. $\mathrm{H}$. Wu et al [8] and Ziouva et al [9] complete the CSMA/CA protocol model of Bianchi, incorporating a maximum number of retransmissions per packet in the backoff algorithm. Y. Xiao [10] improves Ziouva's model by limiting the number of retransmissions and evaluates other performance parameters such as packet drops, packet drop time, throughput limit and saturation delay. The work presented in [11] evaluates the RTS/CTS mechanism comparing it to the basic access mode and presents a method to dynamically modify the contention window to achieve the maximum throughput limit. Y. Xiao et al [12] provide a study of maximum theoretical goodput and delay. J. Jun et al [13] show maximum throughput for IEEE 802.11 networks in absence of errors for different transmission rates and packet sizes.

It is known that voice and video transmission are sensitive to delay and jitter but not so for packet loss as long as losses are less than 5\%, [14]. Data packets on the other side are sensitive to packet loss but not so for delays and jitter. In [15] a study is performed to indicate the maximum amount of voice users that an infrastructure network supports for different voice codecs and includes the importance of asymmetric traffic that the access point must transfer, offering a better model than the ones presented in [16-18]. However, these four studies do not consider changing the access priority for the terminals that compose the wireless network. Y. Lin et al [19] develops an analytical model to obtain the saturation throughput for the IEEE 802.1le [5] protocol which is validated by Ns2 [20]. [5] introduces QoS to wireless networks by differentiating traffic packets and based on this assigns minimum contention windows among other amendments. In a simple scenario it is shown that priority packets may reduce the idle DIFS time, thus increasing their probability of successful transmission.

However, none of the above publications address simultaneously the problem of a network dealing with devices that handle different traffic and packet sizes, maximizing performance by setting RTS/CTS Threshold and contention window parameters for each class of stations when employing legacy IEEE $802.11 \mathrm{a} / \mathrm{b} / \mathrm{g}$ protocols.

\section{IEEE 802.11 Throughput Analysis}

The IEEE 802.11 standard [1] and its subsequent amendments [2-6] have been characterized by having a high overhead. Throughput obtained on the wireless network is a fraction of the operational transmission rates, e.g. IEEE 802.11b [2] only achieves a maximum $6 \mathrm{Mbps}$ throughput with a transmission rate of 11 Mbps, the exact result depending on the initial contention window size, employing maximum packet size and assuming that no collisions occur to access the channel. 
IEEE $802.11 \mathrm{a} / \mathrm{g}$ show similar behavior even though using higher transmission rates, which may be analyzed as follows.

The maximum available throughput of the IEEE 802.11 standard variations [1][4] depend on parameter settings defined by each standard. In Table 1 a summary of parameter values for different standard versions are shown. Some parameters are set to default values in wireless devices, while others may be dependent on the environment where the network is being deployed. For instance, if the wireless network is bridged to an Ethernet, packet size is limited to a maximum of 1500 bytes, as Table 1 shows. Actually, the IEEE 802.11 wireless standards have different values, but for analysis purposes it is advisable to use this value since most wireless networks conforming to this standard are bridged to an Ethernet network. Data rates may vary, depending on channel interference or signal to noise ratio. The contention window settings shown are default values, but may be changed in actual devices and the propagation and processing time may vary depending on the distance among devices.

Table 1. Transmission Parameters IEEE $802.11 \mathrm{a} / \mathrm{b} / \mathrm{g}$

\begin{tabular}{cccc}
\hline Parameter & $\mathrm{b}$ & $\mathrm{a} / \mathrm{g}$ & Units \\
\hline Slot time $\sigma$ & 20 & 9 & $\mu \mathrm{s}$ \\
SIFS & 10 & 10 & $\mu \mathrm{s}$ \\
$D I F S$ & 50 & 28 & $\mu \mathrm{s}$ \\
$A C K$ & 14 & 14 & Bytes \\
$C T S$ & 14 & 14 & Bytes \\
$R T S$ & 20 & 20 & Bytes \\
MAC header & 28 & 28 & Bytes \\
PLCP header long & 192 & 20 & $\mu \mathrm{s}$ \\
PLCP header short & 96 & - & $\mu \mathrm{s}$ \\
Maximum Data Packet Size & 1500 & 1500 & Bytes \\
Header rates $R_{H}$ & 1,2 & 6 & Mbps \\
Data rates $R_{D}$ & $1,2,5.5$, & $6,12,18,24$, & Mbps \\
& 11 & $36,48,54$ & \\
$C W_{\min }$ Minimum Contention Window & 32 & 16 & \\
$C W_{\max }$ Maximum Contention Window & 1024 & 1024 & \\
Propagation and Processing Time & 1 & 1 & $\mu \mathrm{s}$ \\
\hline
\end{tabular}

Terminals operating with a DCF CSMA/CA IEEE 802.11 protocol monitor the channel for an idle time, followed by a Distributed Inter-Frame Space (DIFS), and execute the backoff algorithm to send a data frame. The receiver sends an acknowledgement (ACK) after a Short Inter-Frame Space (SIFS) period. After the ACK is received the whole cycle commences again, in which the terminals must wait a DIFS period and a backoff period before transmission.

Goodput is a performance measure that considers the amount of user data transmitted, divided by the time it takes to successfully transmit it. Goodput and throughput are related performance parameters: to obtain throughput from a good- 
put expression simply divide the latter by the data transmission rate. Since IEEE 802.11 standards can be differentiated by transmission rate, we prefer to use goodput as a performance parameter. Ideal goodput can be calculated as shown in equation (1) not taking into account propagation delay and where $L_{\text {data }}$ is the length of data in bits and $T_{\text {succ }}$ is the time required to successfully transmit the data frame. $T_{I}$ is the time the channel remains idle and $T_{\text {coll }}$ is the time involved in collisions. When computing ideal goodput, collisions are disregarded $\left(T_{\text {coll }}=0\right)$ and $T_{l}$ is equal to the median contention window size times the slot times, expressed in equation (3).

$$
S=\frac{L_{\text {data }}}{T_{l}+T_{\text {succ }}+T_{\text {coll }}}
$$

Equation (2) describes the time required for a successful transmission.

$$
\begin{gathered}
T_{\text {succ }}=\frac{2 \cdot P C L P+M A C+A C K}{R_{H}}+\frac{L_{\text {data }}}{R_{D}}+S I F S+D I F S \\
T_{l}=\frac{\left(C W_{\min }-1\right) \cdot \sigma}{2}
\end{gathered}
$$

Combining equations (1), (2) and (3) and replacing parameter values with the ones specified in the standards (a summary of which have been listed in table 1), it is possible to compute maximum goodput for IEEE $802.11 \mathrm{~b}$. For instance, IEEE $802.11 \mathrm{~b}$ networks that transfer packets of 1500 bytes, assuming $R_{H}=1 \mathrm{Mbps}, R_{D}$ $=11 \mathrm{Mbps}$, no collisions, results in an effective goodput of $6.4 \mathrm{Mbps}$. To calculate the goodput for IEEE $802.1 \mathrm{la} / \mathrm{g}$ the equation must be slightly modified to adjust packet sizes with padding to fit to OFDM (Orthogonal Frequency Division Multiplexing) symbol transmission requirements.

Goodput analysis is normally performed for fixed packet sizes, not having in mind that different applications running on the same network may produce different sizes with different delay requirements. To take into account that the network may be serving different applications equation (1) may be modified. Define a group of $d$ (for data) terminals to transmit data packets of size $L_{d}$ at a data transmission rate $R_{d}$ using an initial contention window $C W_{d \min }$ to access the channel utilizing either basic access mode or RTS/CTS. Another group of $v$ (for voice) terminals to transmit data packets of size $L_{v}$ at a data transmission rate $R_{v}$ using an initial contention window $C W_{\text {vmin }}$ to access the channel. To develop an ideal goodput expression, consider that on average all terminals configured with $C W_{d m i n}$ access the channel during a transmission cycle once. For arbitrary reasons $C W_{v \min }$ will be equal or less than $C W_{d m i n}$. We will later learn that in order to favor access to real time applications like voice, for instance, this will be a convenient setup. Therefore, in a transmission cycle $d$ transmissions will take place, while, on the other hand, there will be $v \cdot C W_{d m i n} / C W_{v \min }$ transmissions of the remaining $v$ terminals. Thus the goodput expression may be written as follows. 


$$
S=\frac{\left(\frac{v \cdot C W_{d \min }}{d \cdot C W_{v \min }}\right) L_{v}+L_{d}}{\left(\frac{v \cdot C W_{d \min }}{d \cdot C W_{v \min }}\right) \cdot\left(T v_{I}+T v_{s u c c}\right)+\left(T d_{I}+T d_{s u c c}\right)}
$$

$T v_{I}$ is the median idle time for the $v$ terminals and $T v_{\text {succ, }}$ and likewise for the $d$ terminals. Thus modifying the value of $C W_{\min }$ for the $v$ and $d$ terminals it is possible to grant higher channel access probability to the terminals that require it.

We would like to point out that the idea of separating goodput expressions according to applications is a powerful concept, because it makes it possible to study the effect of configuration parameter settings on terminals running a specific application, not only to on its own group, but also on the other goodput expressions and the total of the network. This is a concept the authors have not seen in the existing literature and may prove to be quite useful for network administrators.

\section{IEEE 802.11 Goodput with stochastic model}

Similar equations can be developed considering Bianchi's [7] and Wu's [8] approach for stochastic models. Equation (5) shows a generalized formula based on the aforementioned works.

$$
S=\frac{L_{\text {data }}}{T_{l} \cdot P_{l}+T_{\text {succ }} \cdot P_{\text {succ }}+T_{\text {coll }} \cdot P_{\text {coll }}}
$$

$P_{I}$ is the probability that the channel remains idle, $P_{\text {succ }}$ is the probability that during a contention slot a successful transmission occurs and $P_{\text {coll }}$ is the probability that a collision occurs. $T_{\text {coll }}$ is the time required to resolve a collision. This model assumes the channel is error free, collisions among ACK and CTS packages are negligible if RTS/CTS is used, no hidden or exposed terminal is present, fixed size packets are transmitted over the network and terminals operate in saturation to ensure the network may achieve the maximum obtainable goodput. The stationary transmission probabilities of a terminal can be readily obtained from references [7] and [8]. These probabilities only depend on the backoff mechanism of the stations. 


$$
\tau=\left\{\begin{array}{l}
\text { if } \quad r \leq m, \\
\text { if } \quad r>m, \\
\frac{2(1-2 p)\left(1-p^{r+1}\right)}{W\left(1-(2 p)^{r+1}\right)(1-p)+(1-2 p)\left(1-p^{r+1}\right)} \\
2(1-2 p)\left(1-p^{r+1}\right) \\
\left.\hline{ }^{m+1}\right)(1-p)+(1-2 p)\left(1-p^{r+1}\right)+W 2^{m} p^{m+1}(1-2 p)\left(1-p^{r-m}\right)
\end{array}\right.
$$

The probability that a terminal transmits $\tau$, equation (6), depends on the collision probability $p, C W_{\min }$ and the maximum contention window $C W_{\max }$ and the number of retransmissions $r$. The protocol defines that at most 4 retransmissions per packet may take place when operating in basic access mode and 7 in RTS/CTS mode. The number of backoff states $(m)$ depends on $C W_{\min }$ and $C W_{\max }$ given by equation (7).

$$
C W_{\max }=2^{m} \cdot C W_{\min }=2^{m} \cdot W
$$

The probability that a station collides $(p)$ is given by equation (8).

$$
\begin{aligned}
p & =P(\text { collision } \mid \text { one_terminal_Tx }) \\
& =\left(1-(1-\tau)^{n-1}\right)
\end{aligned}
$$

It is possible to set up an equation system with the relations developed in (6) to (8), thus obtaining the stationary probabilities that a contention slot may stay idle, a successful transmission takes place or a collision occurs. The probabilities are shown in the following equations and combining these results with (6) it is possible to determine the goodput of the system.

$$
\begin{gathered}
P_{l}=(1-\tau)^{n} \\
P_{\text {succ }}=n \tau(1-\tau)^{n-1} \\
P_{\text {coll }}=\left(1-(1-\tau)^{n}-n \cdot \tau \cdot(1-\tau)^{n-1}\right)
\end{gathered}
$$

It is possible to extend this to terminals that transmit different types of traffic as described in the previous section. Equation (6) may be written as the sum of goodputs of 2 different traffic types, (12), which has the advantage of being able to individualize contributions of the overall network performance.

$$
S_{\text {total }}=S_{d}+S_{v}
$$

Where 


$$
\begin{aligned}
S_{d} & =\frac{L_{d} \cdot P d_{\text {succ }}}{T_{l} \cdot P_{l}+T_{S}+T_{\text {coll }}} \\
T_{S} & =T d_{\text {succ }} \cdot P d_{\text {succ }}+T v_{\text {succ }} \cdot P v_{\text {succ }} \\
S_{v} & =\frac{L_{v} \cdot P v_{\text {succ }}}{T_{l} \cdot P_{I}+T_{S}+T_{\text {coll }}} \\
T_{S} & =T d_{\text {succ }} \cdot P d_{\text {succ }}+T v_{\text {succ }} \cdot P v_{\text {succ }}
\end{aligned}
$$

The probability that a $d$ station suffers a collision when it tries to transmit is one minus the probability that no other terminal in the network transmits, equation (15).

$$
\begin{aligned}
p_{d} & =P\left(\text { collision } \mid \mathrm{d} \_ \text {terminal_Tx }\right) \\
& =\left(1-\left(1-\tau_{d}\right)^{d-1}\left(1-\tau_{v}\right)^{v}\right)
\end{aligned}
$$

The same expression may be derived for the $v$ terminals replacing the subscript $d$ by $v$ and vice versa. Equation (15) in conjunction with a modified equation (6), where a probability of transmission is described for $d$ and $v$ terminals, creates a set of 5 equations employing equation (7) as well. The probability of an idle slot is given by equation (16).

$$
P_{I}=\left(1-\tau_{d}\right)^{d}\left(1-\tau_{v}\right)^{v}
$$

$T_{I}$ is equal to a slot time $\sigma$. The probability of a successful transmission of a $d$ terminal is given by the probability that only one $d$ terminal transmits in a time slot and that no other $d$ terminals transmit during this time and also that no $v$ terminals transmit. For a $v$ terminal similar equations are shown in (17) and (18). Time associated to a successful transmission of a $d$ or $v$ terminal is similar to equation (2) but the subscript data of $L_{\text {data }}$ needs to be replaced by $d$ or $v$ to reflect the appropriate payload.

$$
\begin{gathered}
P d_{\text {succ }}=d \cdot \tau_{d}\left(1-\tau_{d}\right)^{d-1}\left(1-\tau_{v}\right)^{v} \\
P v_{\text {succ }}=\left(1-\tau_{d}\right)^{d} \cdot v \cdot \tau_{v}\left(1-\tau_{v}\right)^{v-1}
\end{gathered}
$$

Collision time is expressed by equation (19), where the collision probability is multiplied by the time it takes to resolve that particular collision $T d_{\text {coll }}$ or $T v_{\text {coll }}$ (if it involves two different packet sizes, it will always be the larger packet transmission). These times are identical to a successful transmission times.

$$
\begin{aligned}
T_{\text {coll }}= & \left(1-\left(1-\tau_{v}\right)^{v}\right) \cdot\left(1-\left(1-\tau_{d}\right)^{d}\right) \cdot T d_{\text {coll }}+ \\
& +\left(1-\left(1-\tau_{d}\right)^{d}-d \cdot \tau_{d} \cdot\left(1-\tau_{d}\right)^{d-1}\right) \cdot T d_{\text {coll }}+ \\
& +\left(1-\left(1-\tau_{v}\right)^{v}-v \cdot \tau_{v} \cdot\left(1-\tau_{v}\right)^{n-1}\right) \cdot T v_{\text {coll }}
\end{aligned}
$$


Combining equations (12)-(19) it is possible to model an ad-hoc network for 2 different kinds of traffic.

\section{Saturation Goodput Analysis}

Two models that deliver saturation goodput in presence of different traffic types were developed in the previous section. The stochastic model was contrasted to the work done in [19] obtaining identical results. The model developed in section 2 is very simple but provides a quick way to obtain maximum saturation goodput levels.

Employing the parameters from Table 1 for the IEEE 802.11b standard and configuring the maximum transmission speed for the frame payload and headers, basic access mode, long PLCP and two different packet sizes, it is possible to analyze the effect of varying the $C W_{\min }$ of the different traffic sources on the saturation goodput of the network. All terminals are in the transmission range of each other and may employ the maximum transmission rate to communicate amongst each other.

A real-time application like voice is transmitted by $v$ terminals. Voice packets should have preferences over data packets transmitted by the $d$ terminals to access the channel. The $v$ terminals will transmit packets of $L_{v}=50$ bytes. This packet size is calculated for the G.729A codec, where the voice packet of 10 bytes is encapsulated adding 12 bytes of RTP header, 8 bytes of UDP header and 20 bytes of IPv4 header. Assume data terminals have $C W d_{\min }$ set to the default value of 32 and transmit packets of size $L_{d}=1500$ bytes. To reduce delay times and delay jitter for voice packet transmissions, let us decrease the value of $C W_{\min }$ from the default value of 32 to 16,8 and 4 for the $v$ terminals. The remaining parameters of all terminals are kept at the default values of the IEEE $802.11 \mathrm{~b}$ standard. This should have impact on voice, data transmissions and global saturation goodput, Figure 1 shows a scenario where the number of $v$ terminals is increased from 0 to 10 , the total being always 10 terminals, evaluated for both models.

Figure 1 shows that indeed reducing delays on voice packets by increasing their transmission probability has an effect over both the aggregate goodput of voice and data terminals. As more voice terminals are incorporated into the network the overall goodput of the network decreases and the aggregate goodput of the voice terminals increases. Diminishing the $C W v_{\min }$ grants a higher channel access preference for VoIP packets and this is clearly reflected in both aggregate goodputs of the data terminals and voice terminals. Since smaller VoIP packets have a larger overhead from the MAC IEEE 802.11 layer, their transmission significantly reduces overall goodput of the network. Both figures incorporate a threshold ( $S_{\text {Voice }}$ Minimum) curve that represents the minimum aggregate goodput that G.729A codec require to maintain seamless conversations. Thus the aggregate goodput of the $v$ terminals must be over this threshold to support the required goodput. 


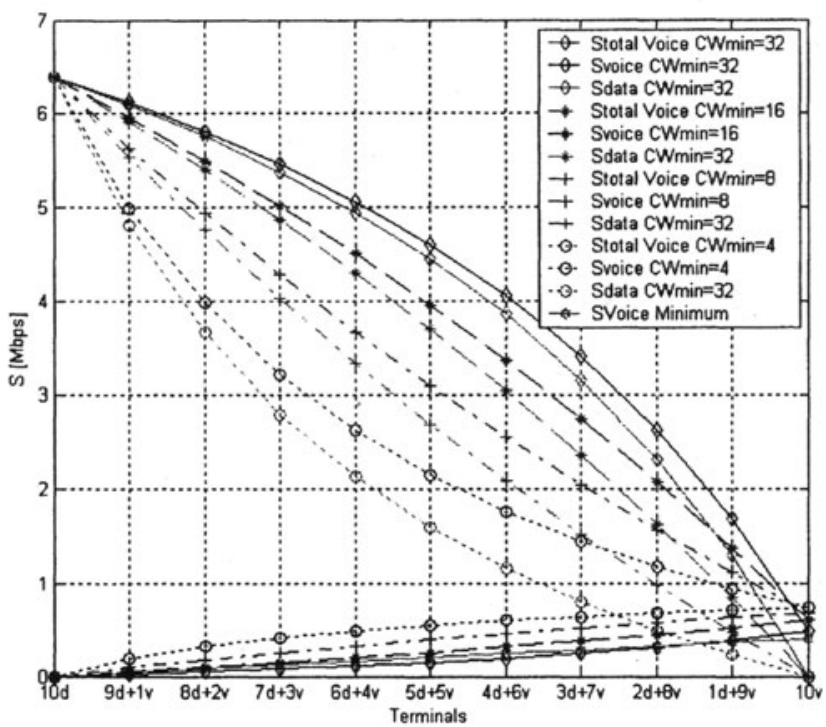

(a)

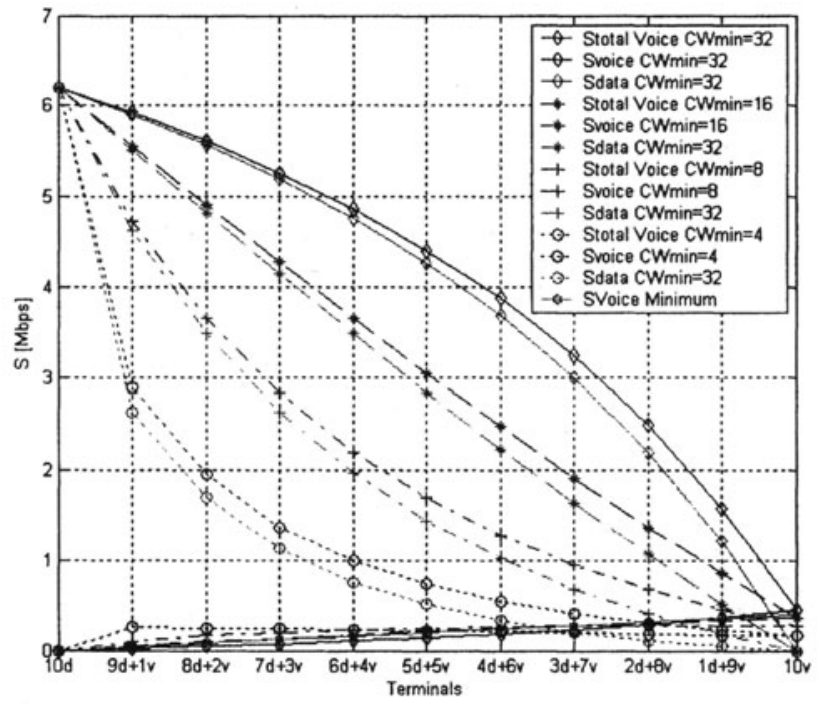

(b)

Fig. 1. Ad-hoc network with 10 terminals varying the amount of $v$ terminal (a) Ideal (Deterministic) model (b) Stochastic model 
When $C W v_{\min }$ is small, it can be clearly seen that the ideal (deterministic) model does not incorporate collisions and provides an optimistic scenario, when compared to the more accurate stochastic model. However it shows clearly the effect of the contention window parameter setting on goodput when dealing with different traffic types. The accuracy of this model when dealing with small initial contention windows and large number of voice terminals is not good as compared to the more accurate stochastic model. Therefore the deterministic model must be used cautiously when these situations arise since this is a clear case when collisions should start occurring.

The selection of $C W v_{\min }$ to improve quality of service for real-time voice station requirements can be done by looking at goodput performance, since higher goodput can be associated to lower delays and delay jitter. Figure 1 shows overall performance, but Table 2 is more accurate than Figure 1, when the decision has to be taken whether the aggregate goodput for voice terminals is sufficient to sustain the minimum required data rate for the vocoder $(40[\mathrm{kbps}])$. In Table 2 aggregate goodput for voice terminals is shown as calculated by applying both models for certain amounts of voice and data terminals and may be compared to the minimum required aggregate transmission rate, $\boldsymbol{S}_{\text {Voice Minimum }}(\mathrm{Codec})$. This value for 3 voice terminals is $120 \mathrm{kbps}$, as Table 2 shows.

Table 2. Goodput values for Voice Terminals from Fig. 1 with different $C W v_{\min }$ values

\begin{tabular}{lccc}
\hline \multicolumn{1}{c}{ Model } & \multicolumn{3}{c}{ Aggregate Goodput for Voice Terminals, [kbps] } \\
\hline \multicolumn{1}{c}{ Number of Terminals } & $7 d+3 v$ & $4 d+6 v$ & $d+9 v$ \\
\hline$S_{\text {Voice Minimum }}$ (Codec) & 120 & 240 & 360 \\
Deterministic $C W v_{\min }=32$ & 77 & 193 & 388 \\
Deterministic $C W v_{\min }=16$ & 144 & 315 & 524 \\
Deterministic $C W v_{\min }=8$ & 254 & 462 & 635 \\
Stochastic $C W v_{\min }=32$ & $\mathbf{7 4}$ & $\mathbf{1 8 4}$ & 365 \\
Stochastic $C W v_{\min }=16$ & 133 & 248 & $\mathbf{3 4 4}$ \\
Stochastic $C W v_{\min }=8$ & 208 & 264 & $\mathbf{2 7 6}$ \\
\hline
\end{tabular}

Table 2 demonstrates that both models predict that the minimum required aggregate goodput is not met when $C W v_{\min }=32$ and there are less then 7 voice terminals, for the codec used in this example. It can be seen that while the minimum aggregate data rate for 3 voice terminals is 120 [kbps], only less than 80 [kbps] can be provided by the network, as calculated by both models. Similarly, when 6 voice terminals are part of the network, the minimum aggregate data rate should be 240 [kbps], but according to both models, aggregate goodput is less than 200 [kbps]. Under these circumstances, selecting $C W v_{\min }=16$ provides the required minimum data rate transfer for the voice codecs (see Table 2), while not deteriorating data packet goodput significantly (see Figure 1). Clearly, when 9 voice terminals are present, the deterministic model does not predict that the aggregate goodput is not enough to sustain seamless voice connections when $C W v_{\min }=8$ or 
16. However it is possible to predict that under default configuration, voice terminals will provide the necessary goodput conditions. This configuration recommendation can only be issued because our approach has been to model both goodputs independently and not as a whole, as most approaches do.

Another interesting aspect is that the configuration recommendations obtained applying the deterministic (collision free) model predict are quite similar to the more precise stochastic model, when the number of voice terminals is not large, although overall goodput values are overestimated. This result has not been reported in the literature, as far as the authors know, and constitutes valuable information for network administrators, since the deterministic model is fairly simple to compute, as compared with the more accurate stochastic model.

\section{Conclusions}

The migration to technologies that rely on IP networks to transmit media rich content demand assigning priority to applications that cannot accept significant delays. Increasingly, wireless networks intended to provide data terminal connections are being used to transmit other kind of information. Therefore it will be relevant to be able to tune configuration parameters of the network devices to optimize network performance using models like the ones proposed in this publication.

Two goodput performance models were presented and compared, a simple one based on stationary behavior of the network, assuming collisions have a negligible effect on network performance, and a second model based on a stationary stochastic model of a network. These models address the problem of a wireless IEEE 802.11 network with devices that generate different traffic patterns (packet sizes and delay constraints), making it possible to analyze goodput performance either for the total network or a specific group of terminals. Parameter settings of the existing standards, like RTS/CTS Threshold, and initial (and maximum) contention window for classes of stations handling traffic of different nature - voice, video, data, multimedia and web traffic - can be varied, as well as the number of participating stations in each class, to study the effect on goodput performance. The use of the simpler model may provide a quick - but less accurate - approach to determine the saturation goodput of a wireless network for network administrators work to design and deploy a network. A more elaborate model that models the wireless channel access more accurately under similar conditions was also presented.

The fact that our approach consisted in evaluating goodput independently for parameters running different applications, makes it possible to analyze the effect of configuration parameter settings not only on total goodput, but also for individual applications, thus making it possible to tune these parameters for best performance. This is an approach the authors have not detected in prior publications. 
Due to space limitations we concentrated our analysis on ad-hoc networks, but the extension of this analysis to infrastructure networks is quite straightforward. The application of these models by network administrators is a good practice, especially if the nature of terminals connected to the network, and their traffic patterns are well known. By elaborating on a simple example, we have shown that not always default values of network parameters will provide satisfactory network performance and it pays off to consider changing them to more convenient settings.

\section{Acknowledgement}

This work was supported in part by CONICYT under Grant by project PBCT ACT-11-04 and project UTFSM 23.07.21

\section{References}

1. ANSI/IEEE Std 802.11, Wireless LAN Medium Access Control (MAC) and Physical Layer (PHY) Specifications (1999 Edition)

2. IEEE Std 802.11b-1999, Wireless LAN Medium Access Control (MAC) and Physical Layer (PHY) specifications: High-speed Physical Layer in the $2.4 \mathrm{GHZ}$ Band

3. IEEE Std 802.11a-1999, Wireless LAN Medium Access Control (MAC) and Physical Layer (PHY) specifications: High-speed Physical Layer in the 5 GHZ Band

4. IEEE Std 802.11g-2003, Wireless LAN Medium Access Control (MAC) and Physical Layer (PHY) specifications Amendment 4: Further Higher Data Rate Extension in the $2.4 \mathrm{GHZ}$ Band

5. IEEE Std. 802.11e, Wireless medium access control (MAC) and physical layer (PHY) specifications: Medium access control (MAC) enhancements for quality of service (QoS) (November 2005)

6. IEEE P802.11n Draft 1.0, Amendment to STANDARD [FOR] Information TechnologyTelecommunications and information exchange between systems-Local and Metropolitan networks-Specific requirements-Part 11: Wireless LAN Medium Access Control (MAC) and Physical Layer (PHY) specifications: Enhancements for Higher Throughput

7. G. Bianchi, Performance Analysis of the IEEE 802.11 Distributed Coordination Function, IEEE Journal on Selected Areas in Communications, V.18, No.3 (March 2000)

8. H. Wu, Y. Peng, K. Long, and S. Cheng, A Simple Model of IEEE 802.11 Wireless LAN, In Proc. IEEE International Conferences on Info-Tech and Info-net (ICII), Beijing, Vol. 2, pp. 514-519 (October 2001)

9. E. Ziouva, and T. Antonakopoulos, The Effect of Finite Population on IEEE 802.11 Wireless LAN Throughput/Delay Performance, In Proc. $1 l^{\text {th }}$ IEEE Mediterranean Electrotechnical Conference (MELECON), Egypt, pp. 95-99 (May 2002)

10. Y. Xiao, Saturation Performance Metrics of the IEEE 802.11 MAC, In Proc. IEEE Vehicular Technology Conference (VTC) 2003-Fall, pp. 1453-1457 (October 2003)

11. R. Bruno, M. Conti, and E. Gregori, IEEE 802.11 Optimal Performances: RTS/CTS Mechanism vs. Basic Access, In Proc. 13th IEEE Symposium on Personal, Personal, Indoor and Mobile Radio Communications (PIMRC), Lisboa, Portugal, Vol. 4, pp. 1747-1751 (September 2002) 
12. Y. Xiao, and J. Rosdahl, Throughput and Delay Limits of IEEE 802.11, IEEE Communication Letters, Vol. 6, No. 8 (August 2002)

13. J. Jun, P. Peddabachagari, and M. Sichitiu, Theoretical Maximum Throughput of IEEE 802.11 and its Applications, In Proc. of the Second IEEE International Symposium on Network Computing and Applications, Cambridge, pp. 249-256 (April 2003)

14. R. Onvural, Asynchronous Transfer Mode Networks: Performance Issues (2nd Ed, Artech House, 1995).

15. L.X. Cai, X. Shen, J.W. Mark, L. Cai, and Y. Xiao, Voice capacity analysis of WLAN with unbalanced traffic, IEEE Transactions on Vehicular Technology, Vol. 55, Issue 3, pp. 752761 (May 2006)

16. S. Garg, and M. Kappes, An experimental study of throughput for UDP and VoIP traffic in IEEE $802.11 \mathrm{~b}$ networks, In Proc. IEEE WCNC, Vol. 3, pp. 1748-1753 (March 2003)

17. S. Garg, and M. Kappes, Can I add a VoIP call?, Proc. IEEE ICC, Vol. 2, pp. 779-783 (May 2003)

18. D. P. Hole, and F. A. Tobagi, Capacity of an IEEE $802.11 \mathrm{~b}$ wireless LAN supporting VoIP," In Proc. IEEE ICC, Vol. 1, pp. 196-201 (June 2004)

19. Y. Lin, and V.W.S Wong, Saturation throughput of IEEE 802.1 le EDCA based on mean value analysis, IEEE Wireless Communications and Networking Conference 2006, WCNC 2006, Vol. 1, pp. 475 - 480 (April 2006)

20. ns2 Network Simulator; http://www.isi.edu/nsnam/ns/ 